\title{
Antimicrobial Resistance: Genetic Perspectives and Implications
}

\author{
Sarunyou Chusri, M.D., Ph.D. ${ }^{1}$, Apichai Tuanyok, Ph.D. ${ }^{2}$
}

'Division of Infectious Disease, Department of Internal Medicine, Faculty of Medicine, Prince of Songkla University, Hat Yai, Songkhla 90110, Thailand.

${ }^{2}$ Department of Infectious Diseases and Pathology, Emerging Pathogens Institute, University of Florida, Gainesville, Florida 32611, United State of America.

Received 28 February 2018 • Revised 26 July 2018 • Accepted 26 July 2018 • Published online 12 September 2018

\section{Abstract:}

The emergences of antimicrobial-resistant bacteria is raised as a serious global concern. Several interventions are used to solve these issues while those beneficial results are still unclear. The pursuit of knowledge through the nature of antimicrobial resistance seem to be the principal to deal with these problems. Recent researches with advanced technologies elucidate the present characteristics as well as predict their evolutions. Molecular data on developing antimicrobial resistance guides to the appropriate clinical practices and transmission prevention. The plenty data on genetic mobile materials are served to clarify several phenomenon and explain the failure of several interventions for controlling antimicrobial resistance. In this review, we focus on the findings of phenotypic and genotypic data of antimicrobial resistance based established advanced researches. We emphasize to apply of those knowledge to routine practices and policy making for control the current situations antimicrobial resistance.

Keywords: antimicrobial resistance, genetic, implication

Contact: Sarunyou Chusri, M.D., Ph.D.

Division of Infectious Disease, Department of Internal Medicine, Faculty of Medicine, Prince of Songkla University, Hat Yai, Songkhla 90110, Thailand. E-mail: sarunyouchusri@hotmail.com
J Health Sci Med Res 2018;36(4):311-322 DOI: http://dx.doi.org/10.31584/jhsmr.2018.36.4.21 www.jhsmr.org 


\section{Introduction}

\section{Background and significances}

Antimicrobial agents have been used for 70 years to treat patients having infectious diseases. These agents have greatly reduced illness and death among those patients. Because these agents have been used so widely and for so long, their efficacy have become compromised by the emergence of resistance to those anti-bacterial activities. The current drug resistance problem is associated with a wide range of responsible biochemical and physiological mechanisms. The complexity of the processes contributes to the emergence and dissemination of resistance. It is emphasized that the fact we have only basic knowledge on these topics is one of the primary reasons it is significant to achieve the effective prevention and control of resistance development. Although international, national, and local facilities all have recognized this serious problem, and have recommended several guidelines to prevent antimicrobial resistance, the development of antibiotic resistance is still relent. ${ }^{1,2}$

Many of the bacterial pathogens associated with epidemics of human disease have evolved into antimicrobial resistance forms subsequent to antibiotic use. Mycobacterium tuberculosis and Plasmodium spp. are major community-acquired pathogens found in several regions and might become the $21^{\text {st }}$ th-century versions of pathogenic organisms. Other serious infections include hospital-acquired infections ( $\mathrm{HAI}$ ) such as from Acinetobacter baumannii, Burkholderia cepacia, Pseudomonas aeruginosa, Stenotrophomonas maltophilia, Escherichia coli, Klebsiella pneumoniae, Proteus mirabilis, Enterobacter spp., Serratia spp., Clostridium difficile, Enterococcus faecium, Enterococcus faecalis, Salmonella spp., Staphylococcus aureus, Staphylococcus epidermidis, and Streptococcus pneumoniae. Nowadays, many facilities use the term "superbugs" which refers to microbes with enhanced severity due to multiple mutations causing high levels of resistance to the antibiotic classes specifically recommended for their treatment. Then the therapeutic options for these microbes become more limited, and periods of hospital care are more extended and costlier. Furthermore, the extreme resistance can be considered to increase virulence and enhanced transmissibility. ${ }^{3}$

The Centers for Disease Control and Prevention (CDC) estimates that 2 million patients suffer from antimicrobial resistant organisms every year and nearly 100,000 of them die. Furthermore, HAls, mostly due to antimicrobial resistant organisms, result in up to $\$ 4.5$ billion in additional healthcare expenses annually and are responsible for even more deaths when adjusted for admission statistics. However, data on hospital costs in developing countries is still scanty and unclear. ${ }^{4}$

\section{Mechanisms of antimicrobial resistance}

There are many mechanisms that bacteria exhibit to protect themselves from antimicrobial agents, which are classified into several conventional types. Antibiotic modification has been well-established: the resistant bacteria retain the none-modified sensitive target while the antibiotic is inactivated by a produced enzyme or protein. For example, with $\beta$ lactamases, the $\beta$ lactamase enzymatically cleaves the four membered $\beta$ lactam ring, rendering the antibiotic inactive. Most $\beta$ lactamases act to different degrees against both penicillins and cephalosporins; others are more specific, namely, cephalosporinases (for example, the AmpC enzyme found in Enterobacter spp) or penicillinases (found in, Staphylococcus aureus). ${ }^{5}$ Antimicrobial resistant bacteria can protect the target of antibiotic action by preventing the antibiotic from entering the cell or pumping it out faster than it can flow into the target. There are 2 mechanisms include inhibition to access to the cell that depends on the antibiotic, through a water filled hollow membrane protein known as a porin such as imipenem-resistant Pseudomonas aeruginosa due to 
lack of the specific D2 porin, and increased efflux via an energy-requiring transport pump such as fluoroquinolones and aminoglycoside-resistant enterobacteriaceae. ${ }^{6,7}$ The other successful mechanism involves alterations in the primary site of action, meaning that the antibiotic is unable to inhibit the activity of the target because of structural changes in the molecule. For example, Enterococci spp. is regarded as being inherently resistant to cephalosporins because the enzymes responsible for cell wall synthesis known as penicillin binding proteins and most strains of Streptococcus pneumoniae are highly resistant to penicillins due to acquiring DNA from other bacteria, which changes the enzyme so that they develop a low affinity for penicillins. ${ }^{8}$ The final mechanism by which bacteria may protect themselves from antibiotics is the production of an alternative target (usually an enzyme) that is resistant to inhibition by the antibiotic while continuing to produce the original sensitive target. The appearance in vancomycin-resistant Enterococci has aroused much interest because the genes involved can be transferred to $S$. aureus, and this can thus theoretically result in a vancomycin resistance. The mechanism also represents a variant of the alternative target mechanism of resistance. ${ }^{9}$

\section{Genetic basis of antimicrobiall resistance}

\section{History of molecular antimicrobial resistance}

Since the introduction of the first antimicrobials, sulfonamides, the development of specific mechanisms of resistance has emerged with their therapy. Sulfonamide resistance was first reported early within few years, and the same mechanisms are still operating today. The other major antibiotic story was penicillin, discovered by Alexander Fleming, and 12 years later, several years before the introduction of penicillin as a therapeutic, a bacterial penicillinase was identified. Once this antibiotic became widely used, resistant strains capable of inactivating the drug became prevalent, and synthetic studies were under- taken to modify penicillin chemically to prevent cleavage by several penicillinases. Interestingly, the identification of a bacterial penicillinase before the use of the antibiotic can now be appreciated in the light of recent findings that a large number of antibiotic genes are components of natural microbial populations. In the case of streptomycin, introduced in 1944 for the treatment of tuberculosis, mutant strains of Mycobacterium tuberculosis resistant to therapeutic concentrations of the antibiotic were found to arise during patient treatment. Only in the past few years has been appreciated that gene transmission is a common property of bacteria that has occurred throughout the microbial evolution. The discovery of the presence of putative bacterial gene sequences has heightened awareness of the great importance of horizontal gene transfer in genome evolution. ${ }^{10-12}$

\section{Modern molecular antimicrobial resistance}

The molecular mechanisms of resistance to antibiotics have been explored extensively and have involved widely investigating of the genetics and biochemistry of many different bacterial cell functions. These studies of antibiotic action and resistance have contributed significantly to our knowledge of bacterial structure and function and associated antimicrobial resistance. The resistance processes are broadly distributed in these bacteria and have been well described for a variety of commensals and pathogens; most can be disseminated by several distinct gene transfer materials. Those resistance types that illustrate the difficulties in maintaining effective antibiotic activity in the face of the genetic and biochemical flexibility of bacteria deserve special description. ${ }^{13}$

The genes responsible for $\beta$-lactamase enzymes are the most internationally distributed; the mutations of the genes encoding these enzymes rose to modified catalysts with increasingly broad spectra of resistance. The plasmid-encoded $\beta$-lactamase, TEM, has spawned 
a huge tribe of related families, providing proof of this adaptability. The $\beta$-lactamase genes are ancient and have been found in remote and desolate environments, which imply that novel $\beta$-lactamases with altered substrate ranges occur in the environment. As another example, a new extended-spectrum $\beta$-lactamase (CTX-M) was acquired from environment. This was the first enzyme found to hydrolyze expanded spectrum cephalosporins, especially cefotaxime at a clinically significant level. The CTX-M genes and subsequent variants are highly successful at transmission and are a global phenomenon. Such epidemics of genes with efficient HGT and rapid mutational radiation are next to impossible to control. ${ }^{13,14}$

The recent emergence of a novel fluoroquinolone (FQ) resistance mechanism is one of the interesting genetic juggleries. A few experts have predicted that resistance to this new class of DNA gyrase inhibitors is unlikely, since at least two mutations would be required to generate a significant resistance phenotype prior to introducing these agents into clinical practice. Initially, it was also expected that horizontally transmitted $F Q$ resistance was unlikely to occur otherwise not encountering mutants of the target bacterial gyrase genes and efflux. More unexpectedly, a transmissible mechanism of $F Q$ inactivation has made its appearance after exposure of many aminoglycosides then $\mathrm{N}$-acetyltransferases (primarily related to exposure to several aminoglycosides). They have developed the capacity to modify a secondary amine on the FQs, leading to resistance. The latter story includes the impact of a low-level tolerance that favors the selection of resistance mutations. Another unpredicted FQ resistance mechanism is known as Qnr, a widespread family of DNA-binding proteins, which are responsible for low levels of quinolone resistance. Another important story is macrolide resistance, which began with the failure of erythromycin and those successors, were firstly concerned with the problem of methicillin resistant organisms such as
MRSA and MRSE. It has been generally recognized that strains resistant due to a number of different mechanisms are now globally spreading. Macrolides act by binding at different peptide binding sites of the $50 \mathrm{~S}$ ribosome subunit. Resistance can occur by modification of these subunits of RNA or components of the binding site..$^{15,16}$

\section{Genetic materials for antimicrobial resistance}

\section{Resistome}

Risistome is the diversity and dynamic of the antibiotic resistance genes that are harboured by the microbiota. Several bacterial strains can be resistant to antibiotics without any antibiotic pressure. Those can be isolated from their environment and planted on antibiotic-containing media in the laboratory. It is not surprising that most possess genes encoding resistance to antibiotics they produce. In several cases, the resistance mechanisms have been identified and shown to be specific enzymatic modifications of the antibiotics. For example, Streptomycetes have produced a variety of $\beta$-lactamases even if these organisms have been never exposed to any $\beta$-lactam antibiotics. In other cases, resistance of producing organisms to their products has been identified as due to efflux systems as well as multiple mechanisms of resistance, as found in the tetracycline producer Streptomyces spp. which are frequent in enzyme producing bacteria. A significant number of strains were naturally resistant to an average of 7 or 8 antibiotics. The population of resistant genes in nature is referred to as the environmental antibiotic resistome. This is the best evidence available for the presence of a major environmental pool of genes with the potential to be captured and expressed as resistance to human antibiotics. ${ }^{17-19}$

\section{Subsistome}

The 'subsistome' refers to a subset of genes in the resistome that permits microbes to degrade antibiotics 
and use them as an energy source. Obviously, catabolic pathways responsible for antibiotic digestion in nature provide a rich source of potential resistance determinants; additional studies should reveal novel mechanisms of resistance to most antibiotic classes. The finding from previous studies on the survival of bacteria with co-existing of potential organisms sourcing protein material supposed subsistome including proteobacteria, Burkholderia spp. and Pseudomonas spp. More recently, the demonstration that a proportion of the microbes in the environment are capable of metabolizing antibiotics has provided additional evidence for the existence of enzymatic mechanisms for the biodegradation of antibiotics that enable bacteria to subsist on antibiotic substrates. This work has revealed the presence of a great diversity of antibiotic-modifying enzymes in nature, and these enzymes provide the basis for a wide range of antibiotic resistance mechanisms. ${ }^{20,21}$

\section{Microbiome}

There is increasing evidence that bioactive agents as antibiotics or similar compounds play roles as chemical signals. Based on estimates of the putative small molecule biosynthetic clusters predicted from the nucleotide sequences of bacterial genomes, most human ecological and other environmental microbes have the genetic capacity to produce a number of bioactive compounds that are active at very low concentrations. Since many human floral bacteria have the capacity to produce up to 20 different bioactive compounds, the notion of community structures controlled by small molecules becomes obvious. Although these compounds may have antibiotic activity at high and often non-physiological concentrations, their functions as chemical signals or modulators, as with the well-known quorum-sensing auto inducers, may be paramount on human flora. To date, microbiome data are obtained predominantly in three forms: (1) 16S rRNA gene sequence surveys that provide a view of microbiome membership, (2) metagenomic data used to portray functional potential, and (3) metatranscriptomic data to describe active gene expression. ${ }^{22,23}$

The human microbiome consists of unicellular microbes - mainly bacteria occupying nearly every surface of our bodies - and has been linked to a wide range of phenotypes in health and disease. High-throughput assays have offered the first comprehensive culture-free techniques for surveying the members of these communities and their bio-molecular activities at the transcript, protein, and metabolic levels. Increasing understanding of the natural biological activities of microbes may provide novel methods for indispensable therapeutic options. ${ }^{24,25}$

\section{Parvome}

Recently, the significance of the roles of many millions of low-molecular-weight organic compounds on antimicrobial resistance, are produced by not only bacteria, but also other microbes and plants. Those productions require well-defined biochemical pathways and involve biosynthetic gene clusters that are frequently larger than $100 \mathrm{~kb}$. Some researchers coined the word "parvome" for this group of organisms, a combination of parv- (Greek prefix: small) and -ome (Latin suffix: group). The discovery guides ecological perspectives on antimicrobial resistance. The remarkable points are demonstrated the origins of some of the small bioactive organic molecules involved in antibiotics and the natural roles of those compounds. The structural components of antibiotics appear to have existed in the biosphere for billions of years, as evidenced by the number of primordial amino acid derivatives (many of them components of non-ribosomal peptides) found in meteorites and byproducts from "prebiotic" reaction conditions. These organisms can be used to explain the discovery of molecules of biosynthetic pathway of erythromycin and streptomycin in fossils at the times of 800 million and 600 million years ago, respectively. ${ }^{26-28}$ 


\section{Genetic evolutions of antimicrobial resis- tance}

\section{Intrinsic resistance}

Intrinsic resistance refers to the primary existence of genes in bacterial genomes that are associated with resistance phenotypes. With the availability mutagenesis techniques and genome sequencing, several intrinsic gene functions in bacteria that may lead to resistance phenotypes in clinical situations have been revealed and extensively explored. As well as phenotypic studies of gene knockout database identifies of specific mutants eliciting activity responses to antibiotic resistance. Several conclusions includes that overexpression of the corresponding wildtype gene could generate a resistance phenotype. Furthermore, prognostic studies have been carried out with a number of pathogenic and floral organisms led to the prediction resistance. Many approaches identify potential resistant genes and provide information on the system's biology of resistance. The techniques of RNA microarray analyses of the effects of antibiotics have provided predictive information including increasing the number of copies of the target genes for an antibiotic can lead to reduction of responsiveness of treatment. ${ }^{29,30}$

\section{Anthropogenic activities induced antimicrobial} resistance

Human activities are generally concerned for antibiotic resistance. An increasing number of antibiotics designed for human applications have been manufactured, used clinically, released into the environment, and widely disseminated, thus providing constant selection and maintenance pressure for populations of resistant bacterial strains in all environments. Since the only available evidence indicates that little in the way of antibiotics is contributed by naturally occurring antibiotic-producing strains in their native environments, it should be assumed that commercial production provides the vast bulk of the antibiotics found in the biosphere. The vast majority of human purposes include growth promotion/prophylactic use in animals, therapeutic/prophylactic use in humans, therapeutic/prophylactic use in aquaculture, therapeutic/ prophylactic use in household pets, pest control/cloning for plants and agriculture and culture sterility, cloning, and selection in research and industry. Those activities significantly trigger the emergence of antimicrobial resistance in both targeted and reservoir organisms. ${ }^{31,32}$

\section{Mobile genetic elements \\ Plasmids}

Plasmids are best thought of as small, auxiliary, dispensable chromosomes. The bacterial plasmids, specifically conjugative plasmids, are able to promote their own transfer and the transfer of other plasmids from one bacterial cell to another. In general, they exist separately from and are replicated independently of the main bacterial chromosomes, although the majority of replication functions are provided by the host cell. Hence, plasmids carry a considerable variety of genes, including those that confer antimicrobial resistance and resistance and virulence determinants. Most plasmids investigated have been circular, double-stranded DNA molecules that range in size from just 2 or 3 genes (2-3 kb) to elements that are equivalent to $10 \%$ or more of the host cell chromosome, accommodating 400 genes or more and encoding up to 4,700 genes. Plasmid-encoded antimicrobial resistance encompasses most if not all classes of antibiotics currently in clinical use and includes resistance to many that are at the forefront of antibiotic therapy. Many resistance plasmids are conjugative, that is they encode the functions necessary to promote cell-to-cell DNA transfer, particularly their own transfer. Conjugative plasmids in Gram-positive bacteria tend to be smaller than those in Gram-negative bacteria, reflecting a different mechanism of cell-to-cell coupling. Conjugation is a replicative process that leaves 
both donor and recipient cells with a copy of the plasmid. Conjugative plasmids can exhibit a broad or narrow host range thus transfer is restricted generally to and between a small numbers of similar bacterial species. A "broad host range' denotes an element able to transfer between widely different bacterial species for example plasmid RP1 (also known as RP4 and RK4), first identified in a clinical strain of Pseudomonas aeruginosa. Indeed, plasmids are common in most bacterial species investigated to date, identifying a large pool of mobile genetic information. ${ }^{33-35}$

\section{Transposons}

Resistant transposons are essentially in-chromosomal movement so are called "jumping” gene systems that relate to antimicrobial resistance genes within the element. There are many forms of transposons distinguished by structure, genetic relatedness and mechanism of transposition. All of these elements have the ability to move both intra- and inter-molecularly, within a DNA molecule or from one DNA molecule to another. These mechanisms generally do not require DNA homology between the element and the sites of insertion and although some transposons show a strong preference for a particular nucleotide sequence at an insertion site, many others show no obvious preference and insert into new sites more or less at random. Transposons encompass small cryptic elements called insertion sequences (IS elements). The transposon differs from an IS element in that it encodes at least one function that changes the phenotype of the cell particularly in terms of antimicrobial resistance. Transposons are either modular systems, referred to as composite transposons, constructed from a pair of IS elements and a central DNA sequence that is not inherently able to transpose, the expression of which alters the cell phenotype or complex systems where transposition and non-transposition functions have not obviously been assembled in a modular fashion. $^{36-38}$ The examples of transposonable elements associated with antibiotic resistances is shown in Table 1.

Table 1 The examples of transposonable elements associated with antibiotic resistances

\begin{tabular}{llll}
\hline Transposon & Size $(\mathbf{K b})$ & Antibiotic resistance & Bacteria \\
\hline Tn1 Tn2 & 4.5 & Beta-lactam & S. aureus \\
Tn5 Tn6 & 3.8 & Kanamycin & E. coli \\
Tn7 & 13.5 & Trimethoprim & E. coli \\
Tn9 & 22.6 & Chloramphenicol & E. coli \\
Tn10 & 8.3 & Tetracycline & E. coli \\
\hline
\end{tabular}

\section{Integrons and gene cassettes}

Bacterial integrons are gene capture systems that utilize site specific recombination, instead of transposition, mechanisms. These elements comprise a specialized recombination system consisting of a gene, a recombination enzyme called an integrase, and a site at which short DNA sequences called gene cassettes. Most resistance integrons conform to a structure known as a class 1 integron. Such elements have a distinctive structure comprising two terminal invariable regions, termed constant sequences (CS), and a highly variable central section. At one end is the $5^{\prime}-\mathrm{CS}$, which accommodates the integron, the cassette insertion site attI and the promoter from which cassette genes are expressed. At the other end is the 3'-CS, which accommodates part of a gene, qacED1, which, when intact, confers resistance to quaternary ammonium compounds, followed by sul, a gene that confers resistance to sulphonamides and two other genes designated as orf5 and orf6. These CS regions enclose a region variable in terms of both length and sequence, which comprises the gene cassettes of the particular integron. This region necessarily varies as the identities 
and number of the gene cassettes change from one integron to another. The complete three-dimensional structure of an integrase has been determined, and the mechanism of resistant gene cassette acquisition is now well understood. Well over 100 cassettes have been identified, covering all the major classes of antibiotics. There is functional and genomic evidence that these elements, long thought to be exclusive to Gram-negative bacteria, are present in Gram-positive bacteria as well. ${ }^{39-41}$

\section{Resistance gene transmission}

The significant accessory genetic elements found in bacteria are capable of acquiring resistant genes and promoting their transmission. Conjugation has been studied extensively, and it has been suggested that frequencies of conjugative transmission in nature are probably several orders of magnitude higher than those under laboratory conditions. Recently, this phenomenon has begun to be explained through powerful studies on microbiomes. With the principal of mechanism for DNA traffic transformation and direct DNA acquisition in the environment, Acinetobacter spp. are naturally competent, and example for frequent HGT. The pathogenic strains typically carry large and multiple genomic islands and thus might be related to environmental roles in the capture and passage of resistant genes. With whole genome sequencing studies, the multiple steps of evolution in the evolution can be demonstrated. ${ }^{42,43}$

Plasmid is the most possible to explain the above phenomenon. Genome sequence analyses of environmental microbes revealed that they are replete with plasmids-mostly large and often carrying multiple genes responsible for antimicrobial resistance. The principal of plasmid overcame the unexplainable existing processes of gene acquisition; transfer, modification, and expression that were in place are expanding and accelerating in the modern biosphere. However, with only concept of plasmid, it cannot be characterized numerous genetic mechanisms implicated in the evolution of antibiotic-resistant populations. The roles phages and transformation is rising and progressively established. This hypothesis can explain the observed enhancing that facilitate DNA exchange, for example, physical proximity by immobilization and promote gene uptake without identification of plasmid. Nowadays, we need to investigate the origins of antimicrobial resistance are to use several methods of developing of restriction enzymes and plasmid cloning techniques. The subsequent extension of pooled bacterial recombinant DNA from plant, animal, and human specimens is still required to elucidate the transmission. ${ }^{44,45}$

\section{Implications}

\section{Antibiotic selection and discovery}

The successful use of any therapeutic agent is compromised by the potential development of resistance to that antibiotic from the first time it is employed. A wide range of mechanisms may be responsible for resistance. Particular case of antimicrobial agents, the complexity of the processes that contribute to emergence and dissemination of resistance cannot be overemphasized, and the lack of basic data on these topics is one of the primary reasons that there has been so little significant achievement in the effective prevention and control of resistance development. It is vital that there should be absolutely no letup in the search for new antimicrobial agents. The microbial parvome is nowhere near being exhausted in the search for new antimicrobials. Current knowledge of inhibitortarget and inhibitor-resistance interactions is not at the point where effective new compounds can be designed or screened with confidence. With increasing application of such interactive genome-associated studies, it can be anticipated that new and valid targets will be identified and tested for inhibitor responses by proper consideration of both the direct and indirect functions of genes. ${ }^{46-48}$ 


\section{Environmental surveillances and metagenomic} analysis

Cloning, PCR, and gene expression techniques have been developed to detect the associated resistant genes from bacterial DNA libraries from clinical specimens and the environment. A potential problem is different gene expressions of the cloned genes in a heterologous host. Taken together with the techniques of whole genome sequencing based on global data, these studies confirm the existence of many potential antibiotic-resistant genes and mechanisms in nature. ${ }^{49}$

Ecological adaptations and environmental management

Some low-molecular-weight natural products have been identified as having a role in antibiotic activity. There are numerous sources of such products, for example from the degradation of natural polymers in nutrient conversions, plant products, and antibiotic compounds. In addition, the environment contains many products that are man-made and/or triggered by human contamination. The major bacterial solution to toxic challenges takes the form of multivalent pumping systems that prevent intracellular accumulation of structurally diverse bactericidal and bacteriostatic substances. With the exception of nonspecific efflux systems, the potential antibiotic resistance determinants found in antibiotic-producing strains are generally associated with structural types or modes of action. It has been suggested that the decontamination of specific substances inhibits the emergence of antimicrobial resistance. However, it is well known that antibiotic activity is only one of the biological properties of bioactive small molecules. ${ }^{50,51}$

\section{Antimicrobial resistance control}

The usual proposals for action to attempt to reduce antimicrobial resistance are strict controls on antibiotic use by humans, requiring accurate prescriptions and controlled therapeutic use in animal husbandry and agriculture. However it is clear that restriction of antibiotic use is difficult to implement on a global scale. Universal adherence to the suggested rules for restraint could have a positive effect, but there were no evidence of elimination of antimicrobial resistance. It can be assumed that a "core" population of antibiotic-resistant strains was established by the in most industrialized nations. Transmission of plasmidencoded resistance mechanisms that developed during that period contributed to international dissemination from those countries. The situation in many developing nations is additional to the complexity with relatively uncontrolled antibiotic use. Commonly used antimicrobials are comparatively inexpensive in these nations. However the common national suggestion regardless the level of development is to restrict antimicrobial use. ${ }^{46}$

The successful chemical modifications of compounds has resulted in active derivatives that are refractory to one or more of the known resistance mechanisms. Even though the targets for resistance function are not modified or removed completely without affecting antibiotic activity. Novel semisynthetic compounds generated by such chemical modifications of antibiotic core structures have extended the useful life of several classes. For example, the structural modification of tigecycline from minocycline to escape the selective efflux pumps which is well-established mechanism of resistance in this class of antibiotic. However, resistant genes evolve in response to new selection pressures, and since multiple mechanisms of resistance exist for every class of antibiotic. Thus the toxicity might be enhanced with the modification. Devising compounds that interfere with the efflux of active inhibitors from the cell is an attractive strategy for the design of modified or combination therapeutics. ${ }^{47}$

Antibiotic cycling is an attempt to reduce selection pressures for resistance and thus prolong the useful life of compounds. Based on the principal of antibiotic 
pressure, this technique does not provide a long-term solution, however, since resistant strains never disappear from the resident population. Thus it may be difficult to decontaminate those antibiotics in the large scale approach. An alternative strategy is combinations of antibiotics that have different modes of action. This combinatorial approach has been used in the past to overcome resistance and has also been applied with success in the treatment. However; detailed pharmacodynamic information is insufficient as well as there being only limited clinical trials. Several strategies for avoiding, inhibiting, or bypassing resistance mechanisms in pathogens have been attempted. Unfortunately, to date, research to extend this approach to other classes of antibiotics has not been successful. ${ }^{48,52}$

Other non-antibiotic approaches for the treatment of bacterial diseases involve stimulation or recruitment of the innate immune system of the host. Recent advances in our understanding of the roles of the human gut microbiome in innate immunity may lead to other therapeutic options. In an ideal world with effective vaccines against all infectious diseases, the use of antibiotics would be reduced drastically. However, despite years of effort, there are few widely used antibacterial vaccines such as the pneumococcal vaccine. ${ }^{53,54}$

\section{Conclusions}

It would be useful that those involved in all efforts to deal with this problem should consider international coordinated multidisciplinary programs with the results of laboratory outputs being deposited in centralized accessible databases to expedite advances in control of infections and its implementation into clinical settings. The future strategies should be based on the advanced genetic data including whole genome sequencing to elucidate the previous findings and guide to next steps of solutions in perspectives of antibiotic resistances. To date the pooled data such as Genebank seem the important resource to access the essential information to resolve these problems. The steadily growing concern over emerging antimicrobial resistance strains in the world would justify the set-up of such databases which would then allow the development of world-wide guidelines for monitoring and recording antibiotic resistance cases around the world. This should provide healthcare experts with appropriate guidelines for appropriate management of bacterial infections and preventing the growth and spread of resistant strains.

\section{References}

1. Aminov RI. A Brief history of the antibiotic era: lessons learned and challenges for the future. Front Microbiol 2010;1:134.

2. World Health Organization. Global action plan on antimicrobial resistance [homepage on the Internet]. Geneva: WHO; 2015 [cited 2017 Jun 15]. Available from: http://www.who.int/iris/ handle/10665/193736

3. World Health Organization. Global strategy for containment of antimicrobial resistance [monograph on the Internet] Geneva: WHO; 2001 [cited 2017 Jun 15]. Available from: http:// www.who.int/drugresistance/WHO_Global_Strategy_English. pdf

4. Organization for Economic Co-operation and Development. OECD health data 2006 [homepage on the Internet]. Paris: OECD; 2006 [cited 2017 Jun 15]. Available from: http:// www.oecd.org/health/healthdata

5. Padmini N, Ajilda AAK, Sivakumar N, Selvakumar G. Extended spectrum $\beta$-lactamase producing Escherichia coli and Klebsiella pneumoniae: critical tools for antibiotic resistance pattern. J Basic Microbiol 2017;57:460-70.

6. Novikova OD, Portnyagina OY, Solov'eva TF. Modified and mutant porins in a study on the molecular basis of nonspecific diffusion. Curr Protein Pept Sci 2017;18:233-9.

7. Chitsaz M, Brown MH. The role played by drug efflux pumps in bacterial multidrug resistance. Essays Biochem 2017;61: 127-39.

8. Hakenbeck R, Brückner R, Denapaite D, Maurer P. Molecular mechanisms of $\beta$-lactam resistance in Streptococcus pneumoniae. Future Microbiol 2012;7:395-410.

9. Courvalin P. Vancomycin resistance in gram-positive cocci. Clin Infect Dis 2006;42(Suppl 1):S25-34. 
10. Swann JP. The search for synthetic penicillin during world war II. Br J Hist Sci 1983;16:154-90.

11. Fletcher C. First clinical use of penicillin. BMJ 1984;289: 1721-3.

12. Wright GD. Antibiotic resistance in the environment: a link to the clinic? Curr Opin Microbiol 2010;13:589-94.

13. Nordmann P. Trends in beta-lactam resistance among Enterobacteriaceae. Clin Infect Dis 1998;27(Suppl 1):S100-6.

14. Paterson DL. Resistance in gram-negative bacteria: Enterobacteriaceae. Am J Infect Control 2006;34(5 Suppl 1):S20-8.

15. Kim ES, Hooper DC. Clinical importance and epidemiology of quinolone resistance. Infect Chemother 2014;46:226-38

16. Correia S, Poeta P, Hebraud M, Capelo JL, Igrejas G. Mechanisms of quinolone action and resistance: where do we stand? J Med Microbiol 2017;66:551-9.

17. Corona F, Blanco P, Alcalde-Rico M, Hernando-Amado S, Lira F, Bernardini A, et al. The analysis of the antibiotic resistome offers new opportunities for therapeutic intervention. Future Med Chem 2016;8:1133-51.

18. Crofts TS, Gasparrini AJ, Dantas G. Next-generation approaches to understand and combat the antibiotic resistome. Nat Rev Microbiol 2017;15:422-434.

19. Hu Y, Gao GF, Zhu B. The antibiotic resistome: gene flow in environments, animals and human beings. Front Med 2017; 11 161-8.

20. Lee PY, Chin SF, Neoh HM, Jamal R. Metaproteomic analysis of human gut microbiota: where are we heading? J Biomed Sci 2017;24:36.

21. Macpherson AJ, de Aguero MG, Ganal-Vonarburg SC. How nutrition and the maternal microbiota shape the neonatal immune system. Nat Rev Immunol 2017;17:508-17.

22. Kim D, Hofstaedter CE, Zhao C, Mattei L, Tanes C, Clarke E, et al. Optimizing methods and dodging pitfalls in microbiome research. Microbiome 2017;5:52.

23. Stone VN, Xu P. Targeted antimicrobial therapy in the microbiome era. Mol Oral Microbiol 2017;32:446-54.

24. Bao Y, Al KF, Chanyi RM, Whiteside S, Dewar M, Razvi H, et al. Questions and challenges associated with studying the microbiome of the urinary tract. Ann Transl Med 2017;5:33.

25. Chotirmall SH, Gellatly SL, Budden KF, Mac Aogain M, Shukla SD, Wood DL, et al. Microbiomes in respiratory health and disease: an asia-pacific perspective. Respirology 2017; 22:240-50.
26. Davies J. How to discover new antibiotics: harvesting the parvome. Curr Opin Chem Biol 2011;15:5-10.

27. Davies J, Ryan KS. Introducing the parvome: bioactive compounds in the microbial world. ACS Chem Biol 2012;7: 252-9.

28. Johnson BK, Abramovitch RB. Small molecules that sabotage bacterial virulence. Trends Pharmacol Sci 2017;38:339-62.

29. Alekshun MN, Levy SB. Molecular mechanisms of antibacterial multidrug resistance. Cell 2007;128:1037-50.

30. Cox G, Wright GD. Intrinsic antibiotic resistance: mechanisms, origins, challenges and solutions. Int $\mathrm{J}$ Med Microbiol 2013;303:287-92.

31. Hernandez J, Gonzalez-Acuna D. Anthropogenic antibiotic resistance genes mobilization to the Polar Regions. Infect Ecol Epidemiol 2016;6. DOI: 10.3402/1ee.v6.32112

32. Tripathi V, Cytryn E. Impact of anthropogenic activities on the dissemination of antibiotic resistance across ecological boundaries. Essays Biochem 2017;61:11-21.

33. Filutowicz M, Burgess R, Gamelli RL, Heinemann JA, Kurenbach B, Rakowski SA, et al. Bacterial conjugation-based antimicrobial agents. Plasmid 2008;60:38-44.

34. Williams JJ, Hergenrother PJ. Exposing plasmids as the Achilles' heel of drug-resistant bacteria. Curr Opin Chem Biol 2008;12:389-99.

35. Mathers AJ, Peirano G, Pitout JD. The role of epidemic resistance plasmids and international high-risk clones in the spread of multidrug-resistant Enterobacteriaceae. Clin Microbiol Rev 2015;28:565-91

36. Starlinger P. IS elements and transposons. Plasmid 1980;3: 241-59.

37. Canica M, Manageiro V, Jones-Dias D, Clemente L, GomesNeves E, Poeta P, et al. Current perspectives on the dynamics of antibiotic resistance in different reservoirs. Res Microbiol 2015;166:594-600.

38. Pagano M, Martins AF, Barth AL. Mobile genetic elements related to carbapenem resistance in Acinetobacter baumannii. Braz J Microbiol 2016;47:785-92.

39. Deng Y, Bao X, Ji L, Chen L, Liu J, Miao J, et al. Resistance integrons: class 1, 2 and 3 integrons. Ann Clin Microbiol Antimicrob 2015;14:45.

40. Escudero JA, Loot C, Nivina A, Mazel D. The integron: adaptation on demand. Microbiol Spectr 2015:3. DOI: 10. 1128/microbiolspec.MDNA3-0019-2014. 
41. Gillings MR. Class 1 integrons as invasive species. Curr Opin Microbiol 2017;38:10-15

42. Iredell J, Brown J, Tagg K. Antibiotic resistance in Enterobacteriaceae: mechanisms and clinical implications. BMJ 2016;352:h6420.

43. Al-Tawfiq JA, Laxminarayan R, Mendelson M. How should we respond to the emergence of plasmid-mediated colistin resistance in humans and animals? Int J Infect Dis 2017;54:7784.

44. Hawkey PM. Multidrug-resistant Gram-negative bacteria: a product of globalization. J Hosp Infect 2015;89:241-7.

45. Fernandez-Lopez R, Redondo S, Garcillan-Barcia MP, de la Cruz F. Towards a taxonomy of conjugative plasmids. Curr Opin Microbiol 2017;38:106-13.

46. Pitiriga V, Vrioni G, Saroglou G, Tsakris A. The impact of antibiotic stewardship programs in combating quinolone resistance: a systematic review and recommendations for more efficient interventions. Adv Ther 2017;34:854-65.

47. Nasiri MJ, Haeili M, Ghazi M, Goudarzi H, Pormohammad A, Imani Fooladi AA, et al. New insights in to the intrinsic and acquired drug resistance mechanisms in Mycobacteria. Front Microbiol 2017;8:681.

48. Conlin PL, Chandler JR, Kerr B. Games of life and death: antibiotic resistance and production through the lens of evolutionary game theory. Curr Opin Microbiol 2014;21:35-44.
49. Mancabelli L, Milani C, Lugli GA, Turroni F, Ferrario C, van Sinderen $D$, et al. Meta-analysis of the human gut microbiome from urbanized and pre-agricultural populations. Environ Microbiol 2017;19:1379-90.

50. Wallinga D, Rayner G, Lang T. Antimicrobial resistance and biological governance: explanations for policy failure. Public Health 2015;129:1314-25.

51. Azarbad H, van Gestel CA, Niklinska M, Laskowski R, Roling WF, van Straalen NM. Resilience of soil microbial communities to metals and additional stressors: DNA-based approaches for assessing “stress-on-stress" responses. Int J Mol Sci 2016;17:1.

52. Plantinga NL, Wittekamp BH, van Duijn PJ, Bonten MJ. Fighting antibiotic resistance in the intensive care unit using antibiotics. Future Microbiol 2015;10:391-406.

53. Lipsitch M, Siber GR. How can vaccines contribute to solving the antimicrobial resistance problem? M Bio 2016;7. DOI: 10.1128/mBio.00428-16

54. Kim L, McGee L, Tomczyk S, Beall B. Biological and epidemiological features of antibiotic-resistant Streptococcus pneumoniae in pre- and post-conjugate vaccine eras: a united states perspective. Clin Microbiol Rev 2016;29:52552. 